\title{
Increased mRNA expression of cytochrome oxidase in dorsal raphe nucleus of depressive suicide victims
}

\author{
A Sanchez-Bahillo' \\ V Bautista-Hernandez' \\ Carlos Barcia Gonzalez' \\ R Bañon ${ }^{2}$ \\ A Luna ${ }^{2}$ \\ EC Hirsch ${ }^{3}$ \\ Maria-Trinidad Herrero' \\ 'Clinical and Experimental \\ Neuroscience, Centro de \\ Investigación Biomédica en Red sobre \\ Enfermedades Neurodegenerativas \\ (CIBERNED); ${ }^{2}$ Department of Legal \\ Medicine, Department of Human \\ Anatomy, School of Medicine, \\ University of Murcia, Campus de \\ Espinardo, Murcia 30100 , Spain; \\ ${ }^{3}$ INSERM U679 Hôpital de la \\ Salpêtrière, Boulevard de l'Hôpital, \\ Paris, France
}

\begin{abstract}
Suicidal behavior is a problem with important social repercussions. Some groups of the population show a higher risk of suicide; for example, depression, alcoholism, psychosis or drug abuse frequently precedes suicidal behavior. However, the relationship between metabolic alterations in the brain and premorbid clinical symptoms of suicide remains uncertain. The serotonergic and noradrenergic systems have frequently been, implicated in suicidal behavior and the amount of serotonin in the brain and CSF of suicide victims has been found to be low compared with normal subjects. However, there are contradictory results regarding the role of noradrenergic neurons in the mediation of suicide attempts, possibly reflecting the heterogeneity of conditions that lead to a common outcome. In the present work we focus on the subgroup of suicide victims that share a common diagnosis of major depression. Based on post-mortem studies analyzing mRNA expression by in situ hybridization, serotonergic neurons from the dorsal raphe nucleus (DRN) from depressive suicide victims are seen to over-express cytochrome oxidase mRNA. However, no corresponding changes were found in the expression of tyrosine hydroxylase (TH) mRNA in the noradrenergic neurons of the Locus Coeruleus (LC). These results suggest that, despite of the low levels of serotonin described in suicide victims, the activity of DRN neurons could increase in the suicidally depressed, probably due to the over activation of serotonin re-uptake. No alteration was found in noradrenergic neurons, suggesting that they play no crucial role in the suicidal behavior of depressive patients.
\end{abstract}

Keywords: depression, suicidal behavior, dorsal raphe nucleus, noradrenergic neurons

Suicidal behavior is an important and grave social problem with complex and intricate clinical implications which might constitute the final outcome of several psychiatric conditions (although it may also be an existential choice, in some cases) (Leboyer et al 2005). Among the neurotransmitters associated with neuropsychiatric disorders, noradrenaline and serotonin are closely associated with depression and suicide. The amount of serotonin (5-HT) or its metabolite, 5-hydroxyindoleacetic acid (5-HIAA), is lower in the brainstem and CSF of suicide victims than in normal subjects (Shaw et al 1967; Shaw 1967; Bourne et al 1968; Pare et al 1969; Lloyd et al 1974; Mann et al 2000; Arango et al 1992; Nordstrom and Asberg 1992). In addition, suicidal behavior has been associated with low activity of the serotonin transporter gene (Courtet et al 2001), which could partially explain the high risk of suicidal attempts observed in certain families (Brent et al 2002; Rujescu et al 2007). The noradrenergic system is also thought to be associated with suicidal behavior. Some authors have found that the levels of tyrosine hydroxylase (TH) are increased in neurons from the Locus Coeruleus (LC) of suicide victims (Ordway et al 1994; Ordway 1997) while others have observed that the number of noradrenergic neurons appears to be lower in suicide
Correspondence: Maria-Trinidad Herrero Department of Human Anatomy, School of Medicine, University of Murcia, Campus de Espinardo, Murcia 30100, Spain

Email mtherrer@um.es 
victims and patients suffering major depression (Arango et al 1996). The seemingly contradictory findings in post-mortem studies as regards LC neurons may reflect the heterogeneity of conditions in which suicide victims are exposed.

In the present work, we have focused our investigation specifically on a subgroup of suicidal victims, previously diagnosed with major depression. We analyze the serotonergic and noradrenergic systems measuring, by in situ hybridization, the mRNA expression of cytochrome oxidase (CO), a nonspecific mitochondrial protein that has been widely used as an index of neuronal activity (Herrero et al 1996; Vila et al 1996; Levy et al 1997), in neurons of the dorsal raphe nucleus (DRN) (Jacobs 1986; Birkett and Fite 2005). We also analyze mRNA expression of $\mathrm{TH}$ in LC neurons, which may be a marker of increased neuronal activity of brains from suicide victims and control subjects dying from other causes.

The study was performed in brainstems obtained from six Caucasian subjects (average age, $48 \pm 11$ years) who had been diagnosed with major depression prior to their committing suicide ( 3 males and 3 females) and 5 age-matched controls ( 2 males and 3 females) dying of natural or accidental causes without any cerebral damage.

Brain tissue was obtained from autopsies performed at the Instituto Médico Legal (Murcia Spain). The study was performed according to the policies and ethical rules of the institutions involved. Suicide victims did not present alterations due to abuse of or dependence on psychoactive drugs.

The brainstem was separated and sectioned along the midline, and half of each block was frozen in isopentane with liquid nitrogen and then stored at $-80{ }^{\circ} \mathrm{C}$. Frozen brainstems were sectioned with a cryostat into $20 \mu \mathrm{m}$ sections at $-19^{\circ} \mathrm{C}$ and thaw-mounted on gelatin-coated slides.

Experimental procedures of the in situ hybridization technique and validation of the quantification of the optical density have been described elsewhere (Kastner et al 1994; Herrero et al 1996; Vila et al 1996, 1997; Levy et al 1997). Briefly, slide-mounted sections were dried at room temperature and post-fixed in 3\% paraformaldehyde in potassium phosphate buffer $(\mathrm{pH}=7.4)$ for 30 minutes. Then, sections were treated with $0.25 \%$ acetic anhydride in $0.1 \mathrm{mM}$ ethanolamine $(\mathrm{pH}=8)$ followed by $0.1 \mathrm{M}$ glycine and dehydration in increasing concentrations of ethanol. Sections were then incubated for 3.5 hours at $50{ }^{\circ} \mathrm{C}$ in a moisture chamber in hybridization solution, containing the antisense ${ }^{35} \mathrm{~S}$-Labelled RNA probe (either $\mathrm{CO}$ or $\mathrm{TH}$ ). After post-hybridization, sections were treated with formamide and RNAse, and then rinsed in $2 x \mathrm{SSC}$, dehydrated in increasing solutions of ethanol, delipidated in xylene and air dried. Then, sections were coated with nuclear tract emulsion (Kodak NTB2), exposed for 4 weeks until development, counterstained with Nissl, delipidated and cover-slipped with Ralmount (BDH Ltd, Poole, Dorset, England) (Kastner et al 1994; Herrero et al 1996; Vila et al 1996, 1997). TH and CO mRNA expression were measured quantifying the optical density of silver grains of each neuron in the LC and DRN, respectively. Quantification of the optical density was performed under bright field microscope light with a 40x objective (Nikon) defining the perimeter of each neuron and using computer software (Scion Image) as described previously (Herrero et al 1996; Barcia et al 2004). Data were expressed as mean \pm SEM. Differences in mRNA expression between suicide victims and control subjects were analyzed by Student t-test.

Quantitative analysis revealed a statistically significant increase in $\mathrm{CO}$ mRNA expression in the DRN of suicide victims compared with controls subjects. In contrast, TH mRNA expression in LC showed no statistically significant difference between suicide victims and control subjects (Figure 1).

The increase in CO mRNA expression observed in the DRN of suicide victims is indicative of unspecified physiological activity of the serotonin neurons, in apparent contradiction with the gener ally accepted finding of decreased serotonin content (Bourne et al 1968; Arango et al 1992; Nordstrom and Asberg 1992). However, post-mortem studies of suicide victims, using radio-ligand binding to quantify different types of 5-HT receptors, have demonstrated a significant increase in $5-\mathrm{HT}_{1 \mathrm{~A}}$ auto receptors in serotonergic neurons from the DRN (Stockmeier et al 1998). The increased activity of DRN neurons reported in the present work may be related with the described increase of 5-HT $\mathrm{HA}_{1 \mathrm{~A}}$ receptor production (Stockmeier et al 1998). Since serotonin levels are generally decreased in the brain of suicide victims (Shaw et al 1967; Shaw 1967; Bourne et al 1968; Pare et al 1969; Lloyd et al 1974; Arango et al 1992; Nordstrom and Asberg 1992; Mann et al 2000), the enhanced activity of DRN neurons might result in an increased energy demand, accompanied by depletion of the serotonin stores and overactivity of reuptake mechanisms (Stockmeier et al 1998).

The mRNA TH levels in the LC reflect previous results showing no differences in $\mathrm{TH}$ immunoreactivity between depressed suicide victims and normal subjects (Baumann et al 1999). However, these results seem to conflict with previous studies reporting increased TH activity in the LC of suicide victims (Ordway et al 1994; Ordway 1997), although in the latter paper only two suicide victims with a premorbid diagnosis of depression were included, while the diagnosis 

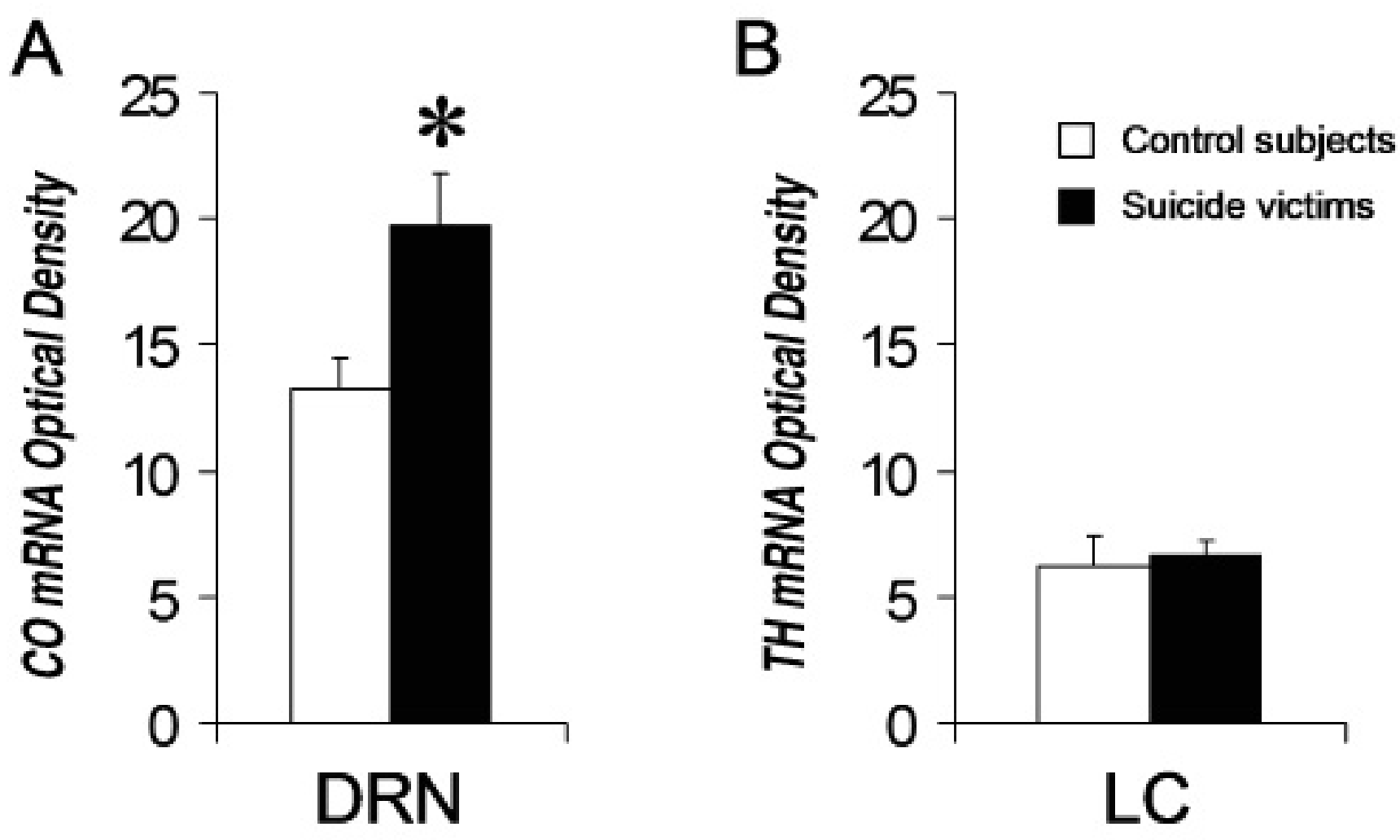

Figure I Quantification of cytochrome oxidase (CO) mRNA expression in neurons of the dorsal raphe nucleus (DRN) (A) and tyrosine hydroxylase (TH) mRNA expression in neurons of the Locus Coeruleus (LC) (B) of depressive suicide victims compared with control subjects. A significant increase in CO mRNA expression was found in neurons from the DRN of suicide victims.

Notes: ${ }^{*} p<0.05$. No statistically significant changes were found in TH mRNA expression between the two groups.

of the other seven suicides in the study group was uncertain. Taken together, these results suggest that there might be a dissociation between depression per se, which may be associated with reduced noradrenergic activity (Baumann et al 1999), and suicidal symptoms, which may be associated with increased activity (Ordway et al 1994), thus masking the effects on the post depression suicide victims.

Further studies are needed to elucidate the role of DRN and LC in suicide victims and how the activation of these nuclei is related with major depression and suicide attempts. Also, further clarification of the differences between different subgroups of suicidal victims needs to be undertaken. Additional in vivo and post-mortem studies of subjects with a high risk of suicide are important in order to determine any clinical and physiological changes and to be able to prevent suicidal behavior.

\section{Acknowledgements}

This work has been supported by grants from: Spanish Ministry of Science (SAF 2002 11675E), Fundación Séneca (FS/05662/PI/07) and CIBERNED (Centro de Investigación Biomédica en Red sobre Enfermedades Neurodegenerativas).
The authors would also like to thank Mr P Thomas for comments and language suggestions on this manuscript. Finally, the authors declare that there is no conflict of interests in the present work.

\section{References}

Arango V, Underwood MD, Mann JJ. 1992. Alterations in monoamine receptors in the brain of suicide victims. J Clin Psychopharmacol, 12:8S-12S.

Arango V, Underwood MD, Mann JJ. 1996. Fewer pigmented locus coeruleus neurons in suicide victims: preliminary results. Biol Psychiatry, 39:112-20.

Barcia C, Sanchez Bahillo A, Fernandez-Villalba E, et al. 2004. Evidence of active microglia in substantia nigra pars compacta of parkinsonian monkeys 1 year after MPTP exposure. Glia, 46:402-9.

Baumann B, Danos P, Diekmann S, et al. 1999. Tyrosine hydroxylase immunoreactivity in the locus coeruleus is reduced in depressed nonsuicidal patients but normal in depressed suicide patients. Eur Arch Psychiatry Clin Neurosci, 249:212-19.

Birkett M, Fite KV. 2005. Diurnal variation in serotonin immunoreactivity in the dorsal raphe nucleus. Brain Res, 1034:180-4.

Bourne HR, Bunney WE Jr., Colburn RW, et al. 1968. Noradrenaline, 5-hydroxytryptamine, and 5-hydroxyindoleacetic acid in hindbrains of suicidal patients. Lancet, 2:805-8.

Brent DA, Oquendo M, Birmaher B, et al. 2002. Familial pathways to earlyonset suicide attempt: risk for suicidal behavior in offspring of mooddisordered suicide attempters. Arch Gen Psychiatry, 59:801-7.

Courtet P, Baud P, Abbar M, et al. 2001. Association between violent suicidal behavior and the low activity allele of the serotonin transporter gene. Mol Psychiatry, 6:338-41. 
Herrero MT, Levy R, Ruberg M, et al. 1996. Consequence of nigrostriatal denervation and L-dopa therapy on the expression of glutamic acid decarboxylase messenger RNA in the pallidum. Neurology, 47:219-24.

Jacobs BL. 1986. Motor activity and the brain serotonin system. Adv Neurol, 43:481-91.

Kastner A, Herrero MT, Hirsch EC, et al. 1994. Decreased tyrosine hydroxylase content in the dopaminergic neurons of MPTPintoxicated monkeys: effect of levodopa and GM1 ganglioside therapy. Ann Neurol, 36:206-14.

Leboyer M, Slama F, Siever L, et al. 2005. Suicidal disorders: a nosological entity per se? Am J Med Genet C Semin Med Genet, 133:3-7.

Levy R, Hazrati LN, Herrero MT, et al. 1997. Re-evaluation of the functional anatomy of the basal ganglia in normal and Parkinsonian states. Neuroscience, 76:335-43.

Lloyd KG, Farley IJ, Deck JH, et al. 1974. Serotonin and 5-hydroxyindoleacetic acid in discrete areas of the brainstem of suicide victims and control patients. Adv Biochem Psychopharmacol, 11:387-97.

Mann JJ, Huang YY, Underwood MD, et al. 2000. A serotonin transporter gene promoter polymorphism (5-HTTLPR) and prefrontal cortical binding in major depression and suicide. Arch Gen Psychiatry, 57:729-38.

Nordstrom P, Asberg M. 1992. Suicide risk and serotonin. Int Clin Psychopharmacol, 6(Suppl 6):12-21.

Ordway GA. 1997. Pathophysiology of the locus coeruleus in suicide. Ann N Y Acad Sci, 836:233-52.
Ordway GA, Smith KS, Haycock JW. 1994. Elevated tyrosine hydroxylase in the locus coeruleus of suicide victims. J Neurochem, 62:680-5.

Pare CM, Yeung DP, Price K, et al. 1969. 5-hydroxytryptamine, noradrenaline, and dopamine in brainstem, hypothalamus, and caudate nucleus of controls and of patients committing suicide by coal-gas poisoning. Lancet, 2133-5.

Rujescu D, Thalmeier A, Moller HJ, et al. 2007. Molecular genetic findings in suicidal behavior: what is beyond the serotonergic system? Arch Suicide Res, 11:17-40.

Shaw DM, Camps FE, Eccleston EG. 1967. 5-Hydroxytryptamine in the hind-brain of depressive suicides. Br J Psychiatry, 113:1407-11.

Shaw RS. 1967. Mental health and suicide. N Engl J Med, 277:378-9.

Stockmeier CA, Shapiro LA, Dilley GE, et al. 1998. Increase in serotonin$1 \mathrm{~A}$ autoreceptors in the midbrain of suicide victims with major depression-postmortem evidence for decreased serotonin activity. J Neurosci, 18:7394-401.

Vila M, Levy R, Herrero MT, et al. 1996. Metabolic activity of the basal ganglia in parkinsonian syndromes in human and non-human primates: a cytochrome oxidase histochemistry study. Neuroscience, 71:903-12.

Vila M, Levy R, Herrero MT, et al. 1997. Consequences of nigrostriatal denervation on the functioning of the basal ganglia in human and nonhuman primates: an in situ hybridization study of cytochrome oxidase subunit I mRNA. J Neurosci, 17:765-73. 